Research Paper

\title{
Cross-talk between MYOC p. Y437H mutation and TGF- $\beta 2$ in the pathology of glaucoma
}

\author{
Yang Yang1,2, AL Sabri Waled Abdulghani Abdulatef ${ }^{1}$, LuSi Zhang'3 ${ }^{3}$ Haibo Jiang', Zhou Zeng', Haibo \\ $\mathrm{Li}^{1,4}{ }^{\bowtie}$, Xiaobo Xia1,4 \\ 1. Eye center of Xiangya Hospital, Central South University, Changsha 410008, Hunan Province, China \\ 2. Department of Ophthalmology, the First People's Hospital of Yueyang, Yueyang, 414000, Hunan Province, China \\ 3. The School of Life Sciences, Central South University, Changsha 410078, Hunan Province, China \\ 4. Hunan Key Laboratory of Ophthalmology, Central South University, Changsha 410008, Hunan Province, China \\ $\triangle$ Corresponding authors: Haibo Li, Email: lihaibo151@aliyun.com and Xiaobo Xia, Email: Xbxia21@163.com. Eye center of Xiangya Hospital and Hunan Key \\ Laboratory of Ophthalmology, Central South University, Changsha 410008, Hunan Province, China. \\ (c) The author(s). This is an open access article distributed under the terms of the Creative Commons Attribution License (https://creativecommons.org/licenses/by/4.0/). \\ See http://ivyspring.com/terms for full terms and conditions.
}

Received: 2020.01.03; Accepted: 2020.04.13; Published: 2020.04.27

\begin{abstract}
Objective: To identify the interaction between the MYOC Y437H mutation and TGF- $\beta 2$ in a family with primary open-angle glaucoma (POAG).

Methods: The MYOC Y437H mutation was identified in a family with POAG; the family was a fourth-generation family with 27 members, of which 6 members were affected. Analysis focused on the secreted myocilin protein and TGF- $\beta 2$ found in the aqueous humor. Samples were taken both from normal controls and MYOC mutant carriers and cross-talk between MYOC Y437H and TGF- $\beta 2$ were evaluated in the trabecular meshwork (TM) cells.

Results: Aqueous humor secreted myocilin protein levels were reduced while TGF- $\beta 2$ levels were increased in patients with the MYOC (c.1309T>C) mutation. This inverse relationship indicated that elevated TGF- $\beta 2$ may be an important pathogenic mechanism in the progression of myocilin-related POAG. In TM cells expressing the MYOC Y437H mutant, exogenous TGF- $\beta 2$ also significantly increased myocilin expression as well as the endoplasmic reticulum (ER) stress markers GRP94 and $\mathrm{CHOP}$. This increase in TGF- $\beta 2$ was also associated with increased cell death in cells carrying the MYOC Y437H mutation.

Conclusion: These data collectively suggest that the mutual interaction between glaucomatous MYOC mutation and TGF- $\beta 2$ contributed to the cell death of TM cells. This relationship also provides a new, therapeutic targets for the treatment of glaucoma.
\end{abstract}

Key words: Glaucoma; MYOC; Aqueous humor; TGF- $\beta 2$

\section{Introduction}

Glaucoma is a kind of optic nerve disease which causes symptoms such as excavation of optic disc and visual field defect. Increased intraocular pressure is a risk factor for developing glaucoma. Critically, it is the second leading cause of blindness in developed countries [1]. Primary open-angle glaucoma (POAG) is the most common type of glaucoma, accounting for $60 \%-70 \%$ of glaucoma cases. In its typical presentation, POAG usually affects both eyes-but not necessarily in a symmetrical way. Regarding its onset, POAG occurs across a wide age range, but is most commonly diagnosed at the age of 40[2]. Important risk factors for POAG include genetics, age, and environment. Previous reports have shown that gene mutations in MYOC, OPTN, and WDR36 are associated with the incidence of POAG [3-5]. Recently, we studied a large family from Hunan in China that had several members suffering from POAG. The family was fourth-generation with 27 members, of which 6 members had POAG. We used PCR and 
direct Sanger sequencing to determine any underlying genetic factors and found that an autosomal dominant gene MYOC (c.1309T $>$ C) mutation was the pathogenic gene for the development of POAG in this family. Furthermore, up-regulation of TGF- $\beta 2$ protein levels was detected in the aqueous humor of patients with this specific MYOC (c.1309T>C) mutation. Finally, we found that exogenous TGF- $\beta 2$ treatment enhanced MYOC Y437H mutation-induced endoplasmic reticulum (ER) stress and resulted in increased cell death. Collectively, these results suggest a molecular mechanism for the cross-talk between MYOC Y $437 \mathrm{H}$ and TGF- $\beta 2$ in the pathology of glaucoma.

\section{Materials and methods}

\section{Study subjects}

A family manifesting the signs and symptoms of POAG was diagnosed at the Xiangya $1^{\text {st }}$ Affiliated Hospital in Changsha, Hunan. The family included 15 males and 12 females, of which four male and two female members were affected with PAOG. All the subjects included in the study signed informed consent approved by Changsha Xiangya Hospital Ethics Committee, and were diagnosed according to the POAG diagnostic criteria drafted in 1987 by Glaucoma Group, Commission of Ophthalmology, Chinese Medical Association.

\section{Clinical examination}

The proband and other members of the family took a physical and ophthalmologic examination, which included visual acuity testing, intraocular pressure (IOP), visual field, slit lamp examination and ultrasound biomicroscopy (UBM).

\section{Mutation detection}

DNA extraction was performed following the standard phenol/chloroform extraction method as previously described [6]. After dissolving, the concentration and purity of gDNA samples were measured using a NanoDrop ND-1000 spectrophotometer (Thermo Scientific, Madison, WI) and underwent appropriate standardization process.

According to the MYOC sequence, Primer 3 online software was used to design the primers. The designed primers were listed in Table 1, and primers were synthesized by Shanghai Sangon Biotech Co., Ltd (Shanghai, China). The PCR system $(10 \mu \mathrm{l})$ was made up of $50 \mathrm{ng}$ DNA template, $30 \mathrm{ng}$ upstream/ downstream primer, $5 \mu$ l doubled Premix Ex Tap TM enzyme (Takara, Japan), and $2 \mu \mathrm{l}$ PCR water. PCR reaction was carried out in a 96-well plate heat circulator (Apllied Biosystem, USA). The reaction conditions used were : i) $95^{\circ} \mathrm{C}$ pre-degeneration for 5 min; ii) $95^{\circ} \mathrm{C}$ degeneration for $30 \mathrm{sec}$; annealing for $58-63^{\circ} \mathrm{C}$ for $30 \mathrm{sec}$ ( 4 primers were completed in the same reaction); extension at $72^{\circ} \mathrm{C}$ for $30 \mathrm{sec}$; total 32circles; iii) extension at $72^{\circ} \mathrm{C}$ for $7 \mathrm{~min}$, and the samples were stored at $4^{\circ} \mathrm{C}$.

The PCR products of the DNA samples collected from the family underwent Sanger sequencing by Boshang Biotech Co., Ltd (Shanghai, China). The sequencing results were analyzed using SeqMan program of DNA STAR software (DNASTAR Inc., Madison, WI) and compared with the reference sequence in the UCSC database to screen for mutations.

Table 1. Primers sequences of MYOC gene

\begin{tabular}{ll}
\hline Primer & Primer sequence $\left(5^{\prime}-3^{\prime}\right)$ \\
\hline MYOC-1F & ctctgtcttccccatgaag \\
MYOC-1R & agcaggtcactacgagccata \\
MYOC-2F & agtgtagtctcggctcacagc \\
MYOC-2R & tctgctcccagggaagttaat \\
MYOC-3-1F & tccgcatgatcattgtctgt \\
MYOC-3-1R & aatgggatggtcagggtctt \\
MYOC-3-2F & catccgtaagcagtcagtcg \\
MYOC-3-2R & tccacaaagttcaaggaaga
\end{tabular}

\section{ELISA, Immunoblotting and Immunostaining}

Aqueous humor was collected and the levels of human TGF- $\beta 2$ were measured using ELISA kit (ab100648, Abcam, Cambridge, MA, UK) according to instructions provided. Immunoblotting and immunostaining were performed as described previously $[7,8]$. The primary antibody used to detect myocilin was obtained from Santa Cruz (sc-137233, Santa Cruz Biotechnology, Santa Cruz, CA), while the transferrin antibody was from Abcam (ab82411). Recombinant human TGF- $\beta 2$ protein was obtained from Thermo Scientific (PHG9124, Thermo Scientific, Madison, WI), dexamethasone was obtained from Sigma (D4902, Sigma, St Louis, MO). The signal intensities of both the myocilin and transferrin bands were analyzed by Image J (National Institutes of Health, Bethesda, MD). The ratio of myocilin relative to the loading control transferrin was first calculated, after which the relative expression was expressed as a percentage of the mean using the control sample as reference.

\section{TM Cell Culture}

Human TM cells were isolated from dissected human TM tissue explants derived from nonglaucomatous donors (NTM). All donor tissues were obtained and managed according to the guidelines in the Declaration of Helsinki for research involving human tissue and also approved by Ethics Committee of Xiangya Hospital, Central South University. Human TM cells were isolated and grown 
as described previously $[9,10]$. Briefly, the TM tissue was dissected and dissociated with $0.05 \%$ Trypsin for $20 \mathrm{~min}$. After centrifugation, the TM cells were grown in DMEM containing $2 \mathrm{mM}$ L-glutamine, penicillin $(100 \mathrm{U} / \mathrm{ml})$, streptomycin $(0.1 \mathrm{mg} / \mathrm{ml})$, and $10 \%$ FBS at $37^{\circ} \mathrm{C}$ in a $5 \% \mathrm{CO}_{2}$ humidified incubator. All of these reagents were obtained from Thermo Scientific.

To construct the vector overexpressing MYOC, a MYOC cDNA (OriGene Technologies, Rockville, MD, USA) was amplified and cloned into lentiviral vector LV6 vector plasmid. The MYOC Y437H mutant vector was generated using a QuikChange ${ }^{\circledR}$ Site-Directed Mutagenesis Kit (Agilent, Santa Clara, CA, USA), and the mutant primer was designed using the QuikChange ${ }^{\circledR}$ Primer Design Program (https://www .genomics.agilent.com/primerDesignProgram.jsp).

Lentiviral supernatant was produced by co-transfection of LV6-WT MYOC or Y437H mutant vector into 293T cells with lentiviral packaging plasmid. For vector transfection, TM cells (passage 3 ) were cultured in $60 \mathrm{~mm}$ dishes to a density of approximately $7 \times 10^{5}$ cells. Cells were then infected for $6 \mathrm{~h}$ with lentiviruses carrying either WT or mutant MYOC, after which the media was changed and culturing occurred for another $5 \mathrm{~d}$. After, all cultures were treated with $50 \mathrm{ng} / \mathrm{ml}$ of TGF- $\beta 2$ for $48 \mathrm{~h}$. All experiments were conducted in triplicate.

\section{Statistical analysis}

All statistical analyses were performed using Prism 5 software (GraphPad, San Diego, CA). Two-tailed Student's $t$ test was used to determine the significance of differences between two groups. All data are presented as mean \pm SEM. Results were considered significant at $p<0.05$.

\section{Results}

\section{Clinical evaluation}

The proband of the family (III:9) was diagnosed with POAG at 16 years of age. After suffering from deteriorating vision in both eyes along with swelling and pain for six months, she visited the hospital to have an ophthalmic evaluation. Visual acuities were $20 / 500$ (0.04) in the right eye (OD) and 20/200 (0.1) in the left eye (OS). IOPs were $25 \mathrm{mmHg}$ in both eyes; cup-to-disc ratio (C/D): OD 0.8, OS 0.9; visual field examination of MD: OD $-32.44 \mathrm{~dB}$, OS $-32.87 \mathrm{~dB}$. The proband was consistent with a POAG diagnosis, given her high IOP, open irido-corneal angle, optic atrophy, and visual field defect (Fig. 1A). UBM detection results are shown in Fig.1B. After admission and IOP stabilization, a left eye combined trabeculectomy was performed under local anesthesia. Post-operative IOP was controlled at approximately $8 \mathrm{mmHg}$. The filtering blebs were functional and the anterior chamber depth was normal. The remaining family members were subjected to ocular examinations, and the clinical characteristics of mutation carriers are listed in Table 2.

\section{MYOC gene mutation screening results}

This particular Chinese pedigree with POAG consisted of 27 members across four generations (Fig.2A). There were no gender difference among the glaucoma patients in the family, which were consistent with its autosomal dominant pattern of inheritance. After detection of the MYOC gene, heterozygosity variation of MYOC: c.1309T $>C$ (P.Tyr437His) was found in members II:3, II:5, II:7, III:5, III:9, and III:10. This mutation was not identified in members III:2, II:9, III:3, III:7, or III:8 (Fig.2B). Given these findings, we considered that the mutation and the disease-related phenotype were co-separated. This mutation was not identified in 100 unrelated normal controls, which excluded the interference of genetic polymorphisms.

Table 2. Clinical Data of $\mathrm{Y} 437 \mathrm{H}$ mutation carriers in this family $(n=7)$

\begin{tabular}{|c|c|c|c|c|c|c|c|c|c|}
\hline $\begin{array}{l}\text { Pedigree } \\
\text { number }\end{array}$ & Gender & $\begin{array}{l}\text { Age at } \\
\text { study } \\
\text { (years) }\end{array}$ & $\begin{array}{l}\text { Highest IOP } \\
\text { NCT OD/OS } \\
(\mathrm{mm} \mathrm{Hg})\end{array}$ & $\begin{array}{l}\text { IOP at study } \\
\text { NCT OD/OS } \\
(\mathrm{mm} \mathrm{Hg})\end{array}$ & $\begin{array}{l}\text { Age at } \\
\text { diagnosis } \\
\text { (years) }\end{array}$ & $\begin{array}{l}\text { Operation } \\
\text { eye/age } \\
\text { (years) }\end{array}$ & $\begin{array}{l}\text { BCVA } \\
\text { OD/OS }\end{array}$ & $\begin{array}{l}\text { C:D ratio } \\
\text { OD/OS }\end{array}$ & $\begin{array}{l}\text { Visual field } \\
\text { damage } \\
\text { OD/OS }\end{array}$ \\
\hline $\mathrm{I}: 1$ & Male & 82 & $48 / 50$ & $33 / 30$ & 39 & $\mathrm{OU} / 40$ & $\mathrm{LP} / 40 \mathrm{~cm}$ & $1.0 / 1.0$ & NA \\
\hline II:3 & Male & 53 & $45 / 48$ & $17 / 14$ & 32 & OU/39 & $\mathrm{HM} / 40 \mathrm{~cm}$ & $1.0 / 1.0$ & NA \\
\hline II:5 & Male & 50 & $39 / 40$ & $16 / 19$ & 28 & OS/34 & $0.2 / 0.8$ & $0.9 / 0.6$ & Severe/Moderate \\
\hline II:7 & Female & 48 & $50 / 53$ & $25 / 20$ & 22 & $\mathrm{OU} / 26$ & $0.5 / 0.6$ & $0.8 / 0.9$ & Moderate/Moderate \\
\hline III:5 & Male & 30 & $42 / 40$ & $12 / 10$ & 23 & $\mathrm{OD} / 27$ & $0.1 / 0.3$ & $0.9 / 0.9$ & Severe/Moderate \\
\hline III:9 & Female & 22 & $44 / 45$ & $13 / 14$ & 16 & OS/20 & $0.04 / 0.1$ & $0.8 / 0.9$ & Severe/ Severe \\
\hline III:10 & Female & 13 & $22 / 23$ & $17 / 18$ & - & - & $1.0 / 1.0$ & $0.2 / 0.2$ & Normal \\
\hline
\end{tabular}

IOP, intraocular pressure; NCT, non-contact tonometer; OD, oculus dexter; OS, oculus sinister; LP, light perception; HM, hand move; BCVA, best corrected visual acuity; C:D ratio, cup/disc ratio; NA, patient was not available for examination 


\section{A OD \\ OS}
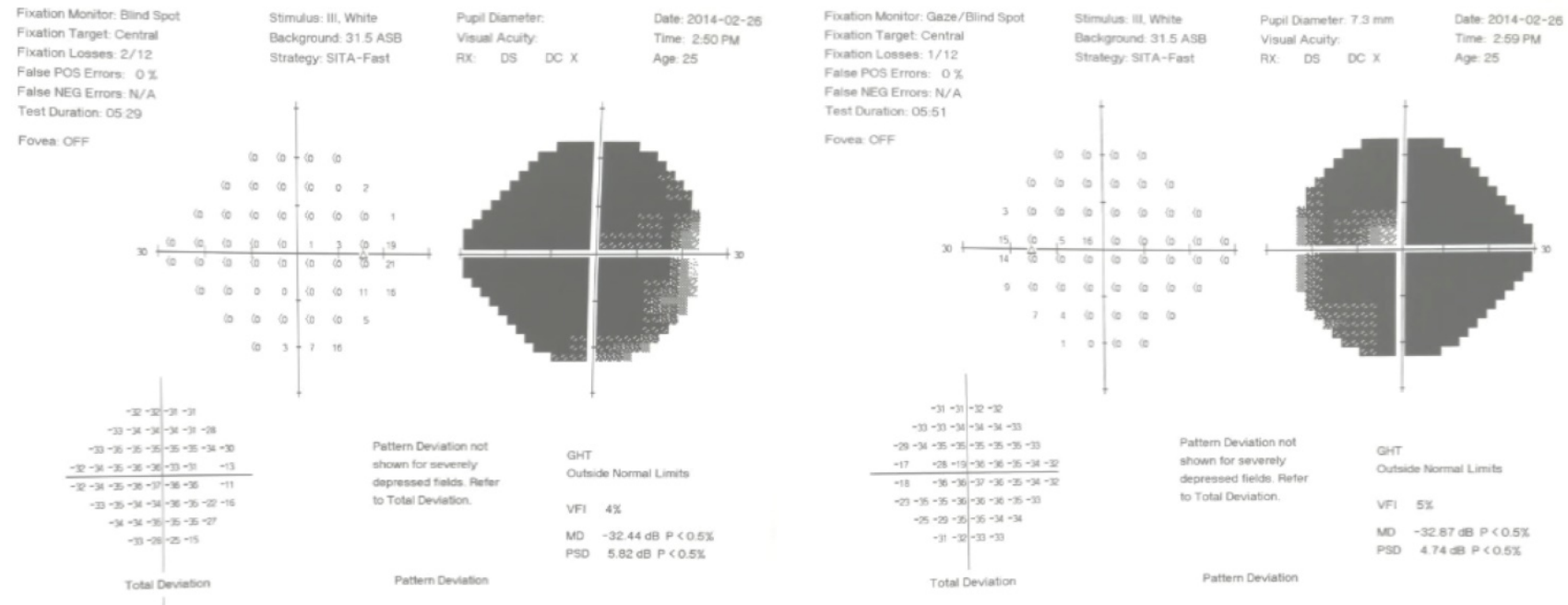

$\mathrm{B}$
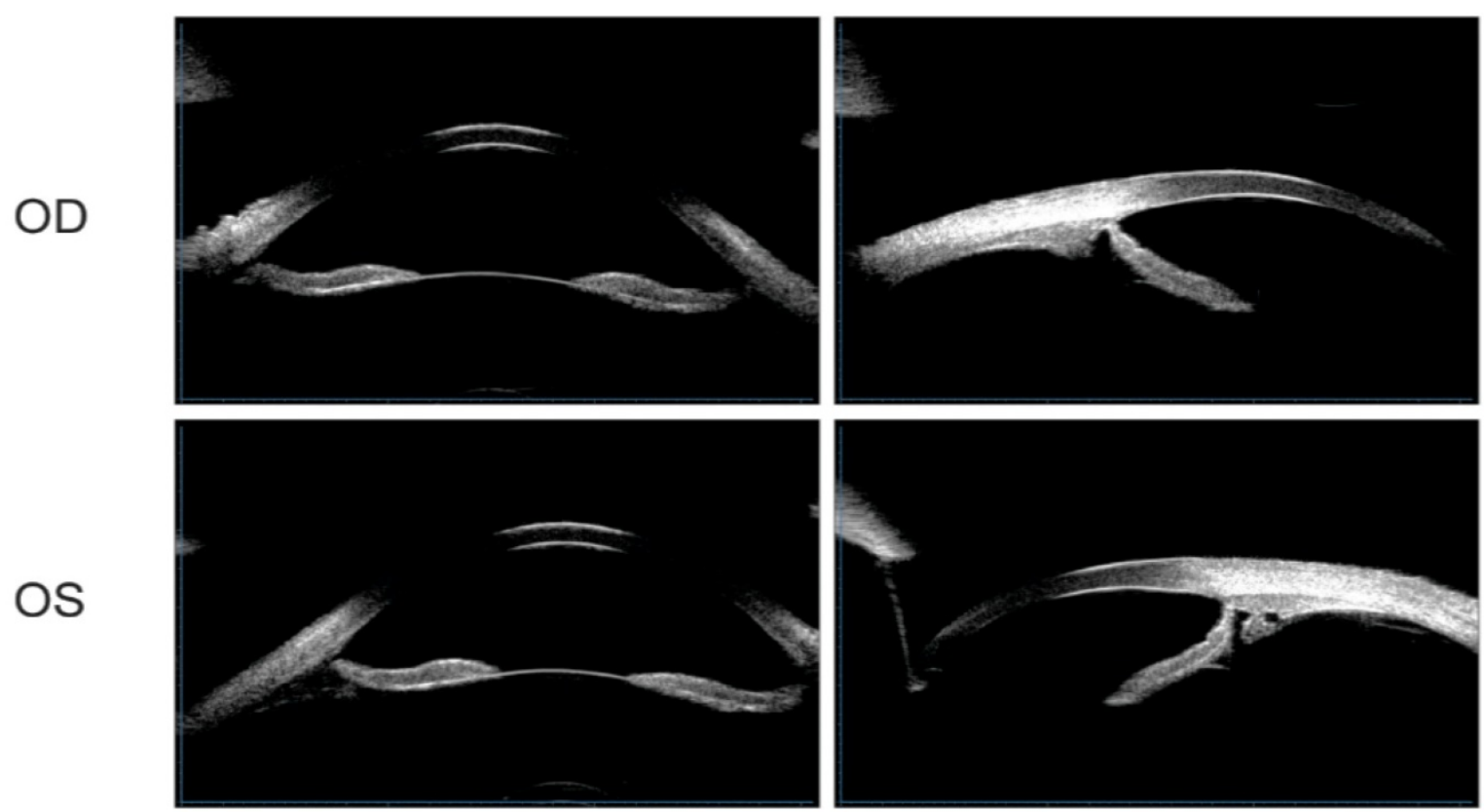

Figure 1. Visual field examination and UBM detection results of the proband. (A) Visual field examination of MD were $-32.44 \mathrm{~dB}$ in the right eye and $-32.87 \mathrm{~dB}$ in the left eye. Note tubular visual field of left eye. (B) The central depth of the anterior chamber was $3.27 \mathrm{~mm}$ in the right eye and $3.25 \mathrm{~mm}$ in the left eye. The iris of both eyes was slightly depressed, and the attachment of the iris root was located at the front without sheltering from the scleral process. The chamber angle was open and ophthalmic lens were in situ.

\section{Aqueous humor TGF- $\beta 2$ levels were elevated in patients with MYOC Y437H mutation}

Myocilin is a secreted protein which can be detected in the aqueous humor. Given this, we next analyzed myocilin protein levels in the aqueous humor of five normal (II:9, III:2, III:3, III:7, III:8) and four MYOC Y437H mutation carriers (II:3, II:5, II:7, III:5) using immunoblotting. In all cases, transferrin was used as the loading control. When compared with normal controls, there was little or no detectable myocilin in the aqueous humor of MYOC Y437H mutation carriers, indicating that myocilin secretion was inhibited by the presence of the MYOC Y437H mutation (Fig.3A). Previous studies showed that cytokines including TGF- $\beta 2$ in aqueous humor have critical roles in patients with open-angle glaucoma [11]. To test whether the MYOC $\mathrm{Y} 437 \mathrm{H}$ mutation was involved in cytokine synthesis, secreted TGF- $\beta 2$ obtained from both the aqueous humor from normal controls and MYOC Y437H mutation carrier patients were tested using a human ELISA kit. When 
compared with the control group, the MYOC Y437H mutants had increased TGF- $\beta 2$ secretion. Specifically, the secreted TGF- $\beta 2$ of the control and MYOC Y $437 \mathrm{H}$ mutation groups were $1053.6 \pm 130.7 \mathrm{ng} / \mathrm{ml}$ and 1582.3 $\pm 174.3 \mathrm{ng} / \mathrm{ml}$, respectively (Fig. 3B). We also found a significant, inverse correlation between myocilin and TGF- $\beta 2$ levels in aqueous humor $\left(\mathrm{R}^{2}=0.62, \mathrm{P}<0.05\right.$, Fig. $3 C)$. The difference in TGF- $\beta 2$ levels in patients with POAG MYOC Y437H mutation might relate to the dysregulated secretion of myocilin in aqueous humor. Taken together, these results provide direct evidence demonstrating that the MYOC $\mathrm{Y} 437 \mathrm{H}$ mutation increased the expression of TGF- $\beta 2$ in aqueous humor, which might be an important pathogenic mechanism in POAG development.

\section{TGF- $\beta 2$ enhances MYOC Y437H mutation induced endoplasmic reticulum(ER) stress}

As the expression of myocilin can be induced by dexamethasone (Dex) in TM cells, we next characterized isolated primary human TM cells by their response to Dex [12]. As given in Fig. 4A, TM cells expressing myocilin at low levels and at high levels after Dex treatment for 5 days. TGF- $\beta 2$ is the major isoform of the TGF- $\beta$ family found in ocular tissue and mediates abnormal extracellular matrix
(ECM) remodeling in TM cells, leading to increased IOP and glaucoma [13]. Previous studies have detected that the MYOC $\mathrm{Y} 437 \mathrm{H}$ mutant was misfolded in the ER, which resulted in ER stress and TM cell death [14]. In order to test whether TGF- $\beta 2$ has effects on MYOC Y437H mutant induced cellular abnormalities, TM cells were infected with adenovirus expressing either WT MYOC or Y437H mutant. These two TM cell groups were then exposed to TGF- $\beta 2(50 \mathrm{ng} / \mathrm{ml})$ for $48 \mathrm{~h}$, after which myocilin expression as well as the expression of two ER stress markers (GRP94 and CHOP) were determined. As shown in Fig. $4 \mathrm{~B}$ and $\mathrm{C}$, myocilin expression was increased in the MYOC Y437H mutant group when compared with TM cells expressing WT MYOC. Furthermore, only expressing of MYOC Y437H mutant, but not WT MYOC, resulted in the elevation of ER stress markers GRP94 and CHOP. Interestingly, exogenous TGF- $\beta 2$ treatment signifycantly increased the expression of myocilin as well as GRP94 and CHOP in TM cells expressing MYOC Y437H mutant. Thus, these data indicated that TGF- $\beta 2$ can enhance the expression of myocilin and ER stress in TM cells expressing MYOC Y437H mutant.

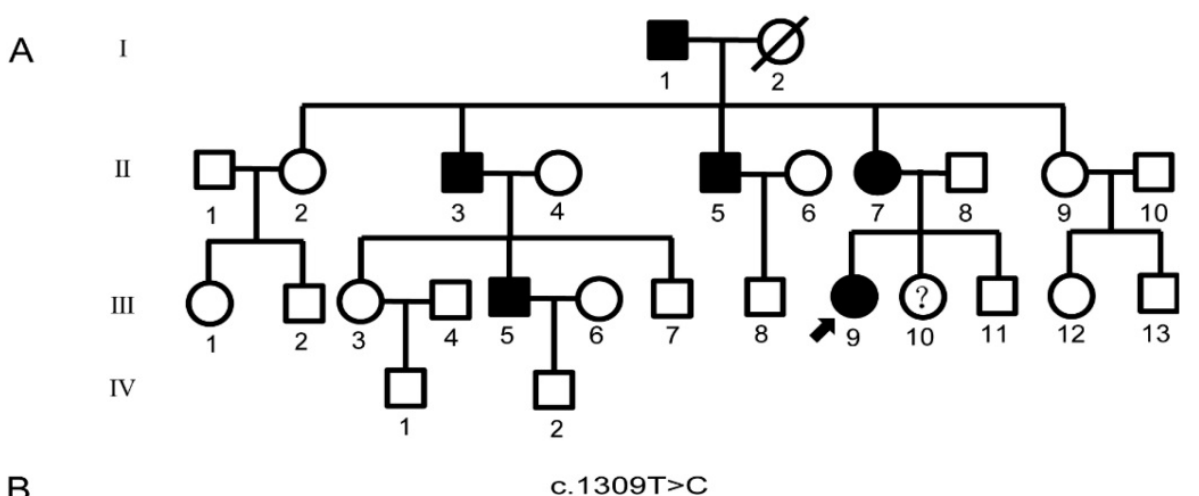

Mutant

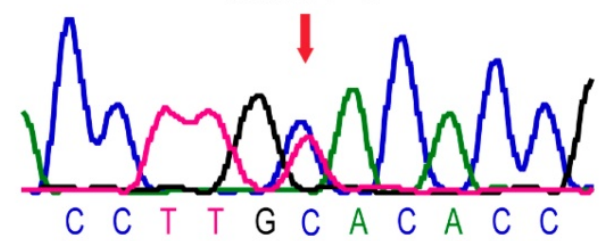

Normal

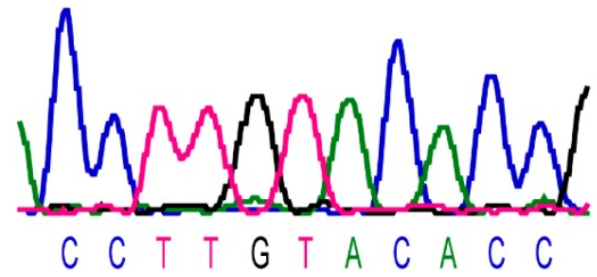

Figure 2. Pedigree structure and sequence diagram of the POAG family. (A) The family with POAG pedigree. Roman numerals referred to generations, and individuals within a generation were numbered from left to right. Proband was noted with arrow. Please note III:10 carried Tyr437His mutation, but was not diagnosed with glaucoma. (B) DNA sequence of MYOC c.1309T>C mutation. Arrows referred to mutant bases. The upper is the sequence map, and the below is the sequence map of the patients, and the red arrow shows the mutation site. 
A

control patient with MYOC $\mathrm{Y} 437 \mathrm{H}$

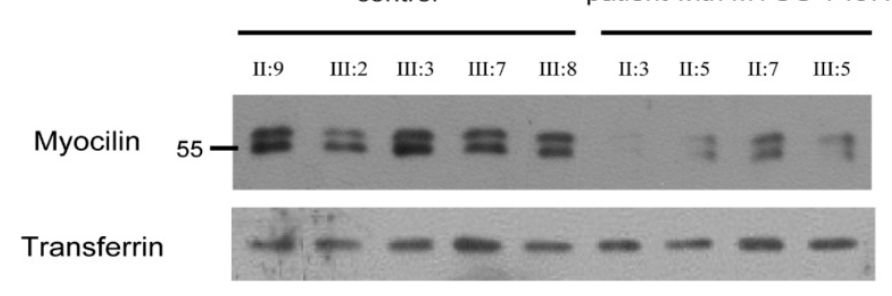

B

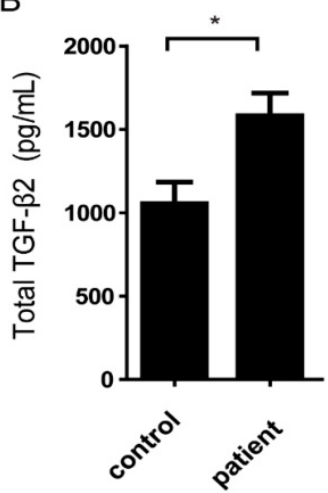

C

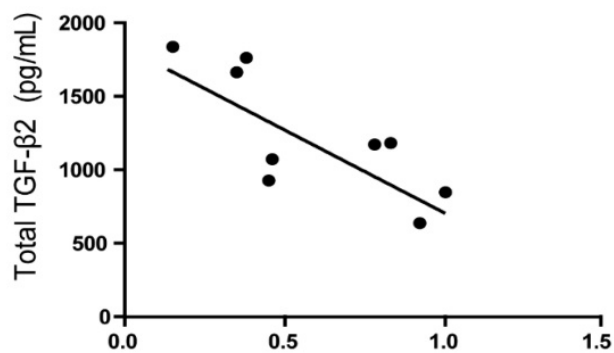

Relative Myocilin expression

Figure 3. Aqueous humor TGF- $\beta 2$ level are elevated in patients with MYOC Y437H mutation. (A) Immunoblotting to detect myocilin and transferrin expression in aqueous humor of normal and MYOC Y 437H mutation carriers. (B) The secreted TGF- $\beta 2$ level in the aqueous humor were detected by human ELISA kit. (C) Pearson correlation analysis of myocilin and TGF- $\beta 2$ expression in aqueous humor. The signal intensities of myocilin and transferrin bands were analyzed by Image J, the relative expression is shown as a percentage of the mean using the control sample as reference.

A

TM

M+Dex
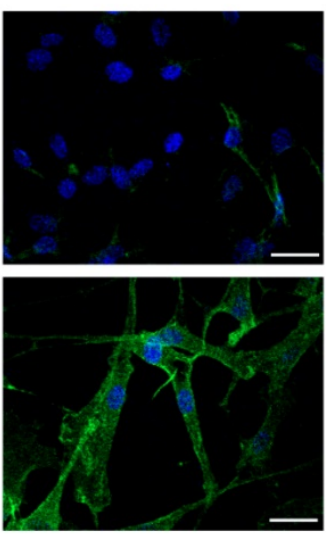

B

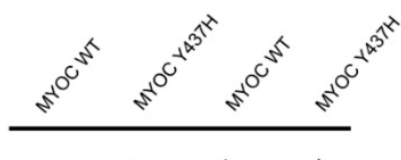

TGF-B2 $(50 \mathrm{ng} / \mathrm{mL})$

Myocilin 55-

GRP94 100-

CHOP

$25-$

GAPDH

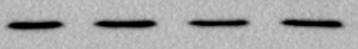

C
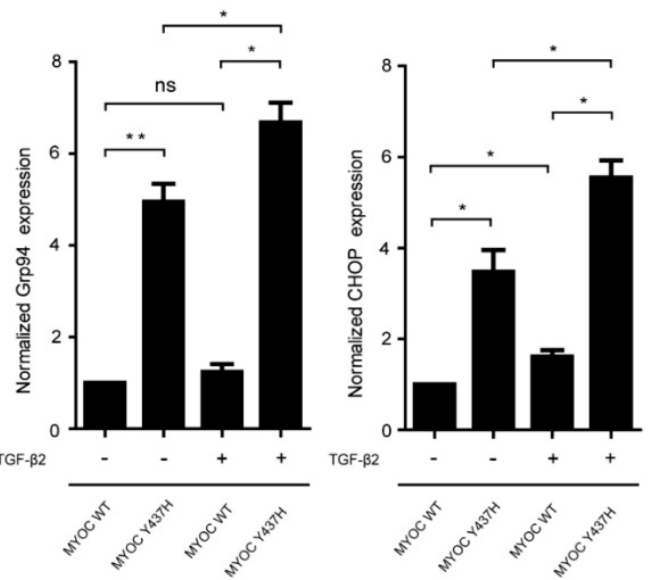

Figure 4. TGF- $\beta 2$ enhances MYOC Y437H mutation induced endoplasmic reticulum(ER) stress. (A) TM cells were exposed to $100 \mathrm{nM}$ Dex for 5 days, then subjected to immunostaining of myocilin. Scale bars $30 \mu \mathrm{m}$. (B) TM cells were overexpressed with WT MYOC or Y437H mutant first, then exposed to TGF- 32 (50ng/ml) for $48 \mathrm{~h}$. Protein from cell lysates were subjected to SDS-PAGE and immunoblotted with the antibodies indicated. (C) Quantification of myocilin, Grp94 and CHOP levels. Relative protein levels were calculated using image J software. The results were obtained from three independent experiments. 
A

MYOC WT
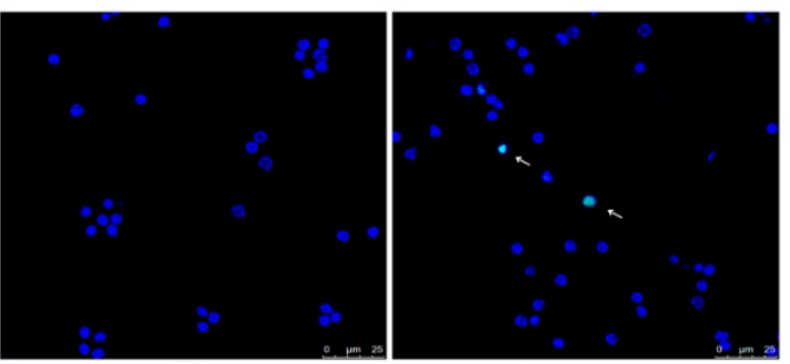

TGF- $\beta 2$
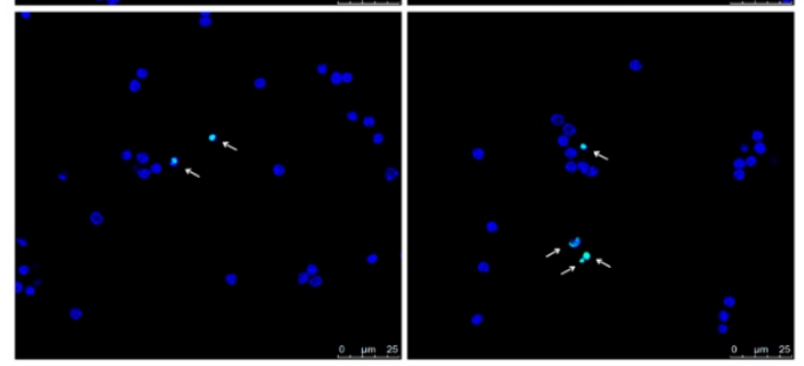

B

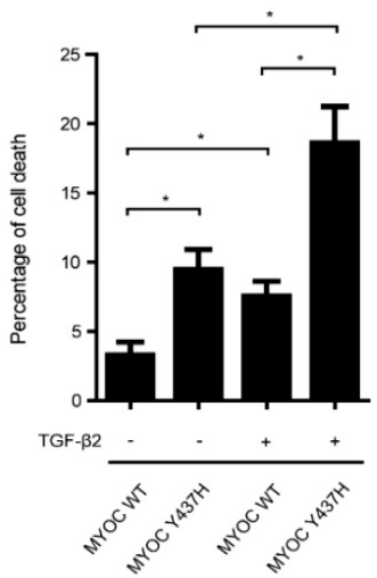

Figure 5. TGF- $\beta 2$ increases MYOC Y437H mutation induced cell death. (A) TM cells were overexpressed with WT MYOC or Y437H mutant first, then exposed to TGF- $32(50 \mathrm{ng} / \mathrm{ml})$ for $48 \mathrm{~h}$. Cell death was detected by TUNEL staining. (B) Quantification of the percentage of TUNEL positive cells.

\section{TGF- $\beta 2$ increases MYOC Y437H mutation induced cell death}

We next sought to determine the interplay between TGF- $\beta 2$ and MYOC Y437H mutation in induced cell death. To do so, human TM cells expressing either WT MYOC or $\mathrm{Y} 437 \mathrm{H}$ mutant were treated with or without TGF- $\beta 2(50 \mathrm{ng} / \mathrm{ml})$ for $48 \mathrm{~h}$, after which TUNEL staining was used to detect cell death (Fig. 5A and B). These results showed that when compared with WT MYOC-expressing TM cells, cells expressing the MYOC Y437H mutant saw a marked increase in cell death. More specifically, the percentage of TUNEL positive cells in these two groups was $3.3 \% \pm 0.9$ and $9.5 \% \pm 1.5$, respectively. Moreover, exogenous TGF- $\beta 2$ significantly increased the percentage of cell death both in the WT MYOC and $\mathrm{Y} 437 \mathrm{H}$ mutant groups (increased to $7.5 \% \pm 1.1$ and $18.6 \% \pm 2.6$, respectively). Taken together, these results demonstrated that TGF- $\beta 2$ enhanced susceptibility to cell death in MYOC Y437H mutant cells.

\section{Discussion}

Glaucoma is an ocular disease with high incidence, unknown etiology, and high genetic heterogeneity. Until now, studies have reported more than 70 MYOC mutation sites in glaucoma patients. The Myocilin protein is encoded by the MYOC gene and is expressed widely in the body, including in skeletal muscle, heart, lung, pancreas, corpus ciliare, trabecular meshwork, and retina [15]. Current international work holds the MYOC mutation rate to be approximately $2-6 \%$ in POAG patients $[5,16]$. The MYOC Y437H mutation is located in the third exon of MYOC and was first reported in 1998 [17]. This study found that the probability of glaucoma in a family carried Tyr437His mutation was about $10^{-12}$ to $10^{-14}$, 
which was far higher than the risk presented in the normal population. Furthermore, the time of glaucoma onset in patients with this Tyr437His mutation was much earlier than in patients with the Gln368X mutation, along with a generally higher corresponding IOP. This phenotype may be related to the dominant negative effect of the MYOC mutation. Additional work showed that the MYOC $\mathrm{Y} 437 \mathrm{H}$ mutant was misfolded and aggregated in the endoplasmic reticulum, inducing abnormal ECM accumulation in the ER and unfolded protein response (UPR) activation [18].

The normal function of the myocilin protein and pathogenic mutations of MYOC in glaucoma is not well understood. In vitro studies have shown that myocilin expression is related to IOP, glucocorticoid, and TGF- $\beta 2[19,20]$, and its expression was related to the synthesis and flow of aqueous humor. Mouse work by Kim et al. found MYOC gene deletion did not change either the intraocular pressure or ocular morphology, which suggested that ability of the MYOC mutation to induce POAG may occur through a gain-of-function effect rather than deficient myocilin expression [4]. MYOC glaucoma-related mutations may activate inflammatory responses by activating the IL-1/NF-kB pathway. This would, in turn, be inhibited by the expression of wild-type MYOC [21]. In this study, we detected a c.1309T>C (p. Y437H) mutation in the MYOC gene in a Chinese POAG family. In this family, we found that although III:10 aged 13 carried the Tyr437His mutation and had high IOP, III:10 was not ultimately diagnosed with glaucoma. This might be because III:10 had not yet reached the age of onset. We further defined decreased myocilin levels and simultaneous increased TGF- $\beta 2$ levels in the aqueous humor of MYOC Y437H mutation carriers. This finding provided a direct link between the MYOC Y437H mutation and TGF- $\beta 2$ production in aqueous humor, which suggests that mutant MYOC $(\mathrm{Y} 437 \mathrm{H})$ protein may activate the inflammatory response in the pathogenesis of POAG by modulating TGF- $\beta 2$.

As the major isoform of TGF- $\beta$ family in ocular tissue, TGF- $\beta 2$ is reported to play important roles in the process of glaucoma via the modulation of ECM synthesis, secretion, and degradation in the human TM cells [22-24]. Previous observations have indicated that TGF- $\beta 2$ concentration is significantly higher in patients with either open-angle glaucoma (OAG) or POAG [11], which mark TGF- $\beta 2$ as a novel therapeutic target for POAG. In fact, targeting TGF- $\beta$ isoforms and/or related receptors has proven promising therapeutic targets in a glaucoma animal model [25]. For instance, in a rabbit model of glaucoma filtration surgery, subconjunctival injection of anti-TGF- $\beta 2$ antibody CAT-152 significantly improved glaucoma surgery outcomes and reduced scarring [26]. However a Phase III study of subconjunctival injection of CAT-152 failed to prevent the failure of primary trabeculectomy [27]. Recently, the first human Phase I trial demonstrated that intravitreal injection of an antisense oligonucleotide ISTH0036 that selectively targets the TGF- $\beta 2$ at the end of trabeculectomy is safe and potentially effective [28]. Collectively, signs of potential therapeutic value have been observed in these studies. However, further research aimed at elucidating the relationship between glaucoma and inflammatory response mediated by TGF- $\beta 2$ are needed, and the results from these studies may help optimize future therapeutic strategies targeting TGF- $\beta 2$.

\section{Abbreviations}

ER: endoplasmic reticulum; POAG: Primary open-angle glaucoma; TM: trabecular meshwork; Dex: dexamethasone; ECM: extracellular matrix; IOP: intraocular pressure; NCT: non-contact tonometer; OD: oculus dexter; OS: oculus sinister; BCVA: best corrected visual acuity; C:D ratio: cup/disc ratio.

\section{Acknowledgements}

We are grateful to our patients and their family and to the normal controls who participated in this study. Haibo $\mathrm{Li}$ and Xiaobo Xia conceived and designed the experiments; Yan Yang and AL Sabri Waled Abdulghani Abdulatef performed the experiments; Haibo Jiang and Zhou Zeng helped to analyze the data; Haibo Li and Xiaobo Xia wrote the paper. Correspondence and requests for materials should be addressed to Haibo Li and Xiaobo Xia.

\section{Funding}

This work was supported by the National Natural Science Foundation of China (NSFC) Grants 81670858 (to Xiaobo Xia), National Natural Science Foundation of Hunan Province, China Grants 2018JJ3817 (to Haibo Li) and the China Postdoctoral Science Foundation Grant 2018M632996 (to Haibo Li). The funders had no role in study design, data collection and analysis, decision to publish, or preparation of the manuscript.

\section{Data Availability}

The data used to support the findings of this study are available from the corresponding author upon request.

\section{Competing Interests}

The authors have declared that no competing interest exists. 


\section{References}

1. Babizhayev MA. Generation of reactive oxygen species in the anterior eye segment. Synergistic codrugs of $\mathrm{N}$-acetylcarnosine lubricant eye drops and mitochondria-targeted antioxidant act as a powerful therapeutic platform for the treatment of cataracts and primary open-angle glaucoma. BBA clinical. 2016; 6: 49-68

2. Quaranta L, Riva I, Gerardi C, Oddone F, Floriani I, Konstas AG. Quality of Life in Glaucoma: A Review of the Literature. Advances in therapy. 2016; 33: 959-81.

3. Kumar S, Malik MA, Goswami S, Sihota R, Kaur J. Candidate genes involved in the susceptibility of primary open angle glaucoma. Gene. 2016; 577: 119-31.

4. Janssen SF, Gorgels TG, Ramdas WD, Klaver CC, van Duijn CM, Jansonius $\mathrm{NM}$, et al. The vast complexity of primary open angle glaucoma: disease genes, risks, molecular mechanisms and pathobiology. Progress in retinal and eye research. 2013; 37: 31-67.

5. Zhou T, Souzeau E, Siggs OM, Landers J, Mills R, Goldberg I, et al. Contribution of Mutations in Known Mendelian Glaucoma Genes to Advanced Early-Onset Primary Open-Angle Glaucoma. Investigative ophthalmology \& visual science. 2017; 58: 1537-44.

6. Zhang L, Liang Y, Zhou Y, Zeng H, Jia S, Shi J. A Missense Mutation in GJA8 Encoding Connexin 50 in a Chinese Pedigree with Autosomal Dominant Congenital Cataract. The Tohoku journal of experimental medicine. 2018; 244 : 105-11.

7. Li Y, Li H, Zhang L, Xiong S, Wen S, Xia X, et al. Growth/differentiation 5 promotes the differentiation of retinal stem cells into neurons via Atoh8. Journal of cellular physiology. 2019; 234: 21307-15.

8. Zhou RR, Li HB, You QS, Rong R, You ML, Xiong K, et al. Silencing of GAS5 Alleviates Glaucoma in Rat Models by Reducing Retinal Ganglion Cell Apoptosis. Human gene therapy. 2019; 30: 1505-19.

9. Wang Y, Osakue D, Yang E, Zhou Y, Gong H, Xia X, et al. Endoplasmic Reticulum Stress Response of Trabecular Meshwork Stem Cells and Trabecular Meshwork Cells and Protective Effects of Activated PERK Pathway. Investigative ophthalmology \& visual science. 2019; 60: 265-73.

10. Keller KE, Bhattacharya SK, Borras T, Brunner TM, Chansangpetch S, Clark $\mathrm{AF}$, et al. Consensus recommendations for trabecular meshwork cell isolation, characterization and culture. Experimental eye research. 2018; 171: 164-73.

11. Agarwal P, Daher AM, Agarwal R. Aqueous humor TGF-beta2 levels in patients with open-angle glaucoma: A meta-analysis. Molecular vision. 2015; 21: 612-20.

12. Xiong S, Xu Y, Wang Y, Kumar A, Peters DM, Du Y. alpha5beta1 Integrin Promotes Anchoring and Integration of Transplanted Stem Cells to the Trabecular Meshwork in the Eye for Regeneration. Stem cells and development. 2020; 29: 290-300.

13. Pervan CL. Smad-independent TGF-beta2 signaling pathways in human trabecular meshwork cells. Experimental eye research. 2017; 158: 137-45.

14. Zode GS, Kuehn MH, Nishimura DY, Searby CC, Mohan K, Grozdanic SD, et al. Reduction of ER stress via a chemical chaperone prevents disease phenotypes in a mouse model of primary open angle glaucoma. The Journal of clinical investigation. 2011; 121: 3542-53.

15. Tomarev SI, Nakaya N. Olfactomedin domain-containing proteins: possible mechanisms of action and functions in normal development and pathology. Molecular neurobiology. 2009; 40: 122-38.

16. Ennis S, Gibson I, Griffiths H, Bunyan D, Cree AJ, Robinson D, et al. Prevalence of myocilin gene mutations in a novel UK cohort of POAG patients. Eye. 2010; 24: 328-33.

17. Alward WL, Fingert $\mathrm{JH}$, Coote MA, Johnson AT, Lerner SF, Junqua D, et al. Clinical features associated with mutations in the chromosome 1 open-angle glaucoma gene (GLC1A). The New England journal of medicine. 1998; 338: 1022-7.

18. Kasetti RB, Phan TN, Millar JC, Zode GS. Expression of Mutant Myocilin Induces Abnormal Intracellular Accumulation of Selected Extracellular Matrix Proteins in the Trabecular Meshwork. Investigative ophthalmology \& visual science. 2016; 57: 6058-69.

19. Patel GC, Phan TN, Maddineni P, Kasetti RB, Millar JC, Clark AF, et al Dexamethasone-Induced Ocular Hypertension in Mice: Effects of Myocilin and Route of Administration. The American journal of pathology. 2017; 187: 713-23.

20. Wu Y, Chen $\mathrm{W}$, Guo $\mathrm{M}, \mathrm{He} \mathrm{Q}, \mathrm{Hu} \mathrm{Y}$. Effects of transforming growth factor-beta2 on myocilin expression and secretion in human primary cultured trabecular meshwork cells. International journal of clinical and experimental pathology. 2014; 7: 4827-36.

21. Itakura T, Peters DM, Fini ME. Glaucomatous MYOC mutations activate the IL-1/NF-kappaB inflammatory stress response and the glaucoma marker SELE in trabecular meshwork cells. Molecular vision. 2015; 21: 1071-84.

22. Takai Y, Tanito M, Ohira A. Multiplex cytokine analysis of aqueous humor in eyes with primary open-angle glaucoma, exfoliation glaucoma, and cataract. Investigative ophthalmology \& visual science. 2012; 53: 241-7.

23. Mody AA, Wordinger RJ, Clark AF. Role of ID Proteins in BMP4 Inhibition of Profibrotic Effects of TGF-beta2 in Human TM Cells. Investigative ophthalmology \& visual science. 2017; 58: 849-59.

24. Wordinger RJ, Sharma T, Clark AF. The role of TGF-beta2 and bone morphogenetic proteins in the trabecular meshwork and glaucoma. Journal of ocular pharmacology and therapeutics : the official journal of the Association for Ocular Pharmacology and Therapeutics. 2014; 30: 154-62.
25. Zhu X, Xu D, Zhu X, Li L, Li H, Guo F, et al. Evaluation of Chitosan/Aptamer Targeting TGF-beta Receptor II Thermo-Sensitive Gel for Scarring in Rat Glaucoma Filtration Surgery. Investigative ophthalmology \& visual science. 2015; 56: 5465-76.

26. Mead AL, Wong TT, Cordeiro MF, Anderson IK, Khaw PT. Evaluation of anti-TGF-beta2 antibody as a new postoperative anti-scarring agent in glaucoma surgery. Investigative ophthalmology \& visual science. 2003; 44: 3394-401.

27. Group CATTS, Khaw P, Grehn F, Hollo G, Overton B, Wilson R, et al. A phase III study of subconjunctival human anti-transforming growth factor beta(2) monoclonal antibody (CAT-152) to prevent scarring after first-time trabeculectomy. Ophthalmology. 2007; 114: 1822-30.

28. Pfeiffer N, Voykov B, Renieri G, Bell K, Richter P, Weigel M, et al. First-in-human phase I study of ISTH0036, an antisense oligonucleotide selectively targeting transforming growth factor beta 2 (TGF-beta2), in subjects with open-angle glaucoma undergoing glaucoma filtration surgery. PloS one. 2017; 12: e0188899. 